\title{
A note on Taylor's hypothesis under large-scale flow variation
}

\author{
M. Wilczek ${ }^{1}$, H. Xu ${ }^{2}$, and Y. Narita ${ }^{3,4}$ \\ ${ }^{1}$ Department of Mechanical Engineering, The Johns Hopkins University, 3400 North Charles Street, \\ Baltimore, MD 21218, USA \\ ${ }^{2}$ Max Planck Institute for Dynamics and Self-Organization (MPIDS), Am Fassberg 17, 37077 Göttingen, Germany \\ ${ }^{3}$ Space Research Institute, Austrian Academy of Sciences, Schmiedlstr. 6, 8042 Graz, Austria \\ ${ }^{4}$ Institut für Geophysik und extraterrestrische Physik, Technische Universität Braunschweig, Mendelssohnstr. 3, \\ 38106 Braunschweig, Germany
}

Correspondence to: M. Wilczek (mwilczek@jhu.edu)

Received: 16 December 2013 - Revised: 17 March 2014 - Accepted: 16 April 2014 - Published: 2 June 2014

\begin{abstract}
Experimental investigations of turbulent velocity fields often invoke Taylor's hypothesis (also known as frozen turbulence approximation) to evaluate the spatial structure based on time-resolved single-point measurements. A crucial condition for the validity of this approximation is that the turbulent fluctuations are small compared to the mean velocity, in other words, that the turbulence intensity must be low. While turbulence intensity is a well-controlled parameter in laboratory flows, this is not the case in many geo- and astrophysical settings. Here we explore the validity of Taylor's hypothesis based on a simple model for the wavenumberfrequency spectrum that has recently been introduced as a generalization of Kraichnan's random sweeping hypothesis. In this model, the fluctuating velocity is decomposed into a large-scale random sweeping velocity and small-scale fluctuations, which allows for a precise quantification of the influence of large-scale flow variations. For turbulence with a power-law energy spectrum, we find that the wavenumber spectrum estimated by Taylor's hypothesis exhibits the same power-law as the true spectrum, yet the spectral energy is overestimated due to the large-scale flow variation. The magnitude of this effect, and specifically its impact on the experimental determination of the Kolmogorov constant, are estimated for typical turbulence intensities of laboratory and geophysical flows.
\end{abstract}

\section{Introduction}

One of the central results from Kolmogorov's celebrated phenomenology (Kolmogorov, 1941) is that the turbulence energy spectrum function takes the form

$$
E(k)=C_{\mathrm{K}} \varepsilon^{2 / 3} k^{-5 / 3}
$$

in the inertial range of scales, that is, the range of scales which is clearly separated from the large, energy-containing scales and the small, dissipative scales. Here $\varepsilon$ is the mean rate of energy dissipation and $C_{\mathrm{K}}$ is often called the Kolmogorov constant. The energy spectrum (Eq. 1) and its onedimensional counterparts have been observed in many laboratory, atmospheric and oceanic flows. Consequently, there is a strong theoretical and practical interest in determining the value of $C_{\mathrm{K}}$, which is assumed to be a universal constant, especially from atmospheric turbulence data (see, e.g., Sreenivasan, 1995).

To measure the energy spectrum without invoking additional hypotheses, multi-point measurements of the turbulent velocity field are necessary. True instantaneous multi-point measurements, however, turn out to be a severe challenge for various reasons. Field measurement techniques, such as particle image velocimetry (PIV), are usually limited to small ranges of resolved scales that do not allow reliable calculation of the energy spectra. For point measurement techniques, such as laser Doppler velocimetry (LDV), acoustic Doppler velocimetry (ADV), ultrasonic anemometry or hotwire anemometry (HWA), many probes have to be used simultaneously to measure the true spectrum, which requires not only high capital investment, but also tedious alignment, 
calibration and maintenance. For intrusive techniques such as HWA, installing many probes in the flow could even result in large disturbances that render the measurements invalid. Moreover, optical-based techniques (PIV and LDV) require uniformly seeded tracer particles for best performance, which is difficult to achieve in geophysical flow measurements. Recent advances in remote sensing provide valuable data on large-scale mean flow fields. The spatial resolutions of these data, however, are still too low to allow quantitative measurement of the energy spectra. Therefore, to date, the energy spectra of geophysical flows are almost exclusively measured with a single probe, and Taylor's frozen turbulence hypothesis is used to reconstruct the spatial variation of the velocity field, which assumes that turbulent fluctuations are carried with the mean flow in a quasi-frozen manner (Taylor, 1938). In other words, instead of spatial variations, experiments usually measure temporal signals that are then mapped to the spatial domain. For the energy spectrum this means that the frequency $\omega$ is related to the streamwise wavenumber $k_{z}$ by the simple relation $\omega=k_{z} U$, where $U$ is the mean velocity of the flow.

The validity of this approximation has widely been studied in various geophysical field and laboratory experiments; some examples include oceanic and surface-water turbulence (MacMahan et al., 2012), atmospheric turbulence (Lappe and Davidson, 1963; Mizuno and Panofsky, 1975; Castro et al., 2011), precipitation field distributions in meteorology ( $\mathrm{Li}$ et al., 2009), wall turbulence (Uddin et al., 1997), and wind tunnel experiments (LeBoeuf and Mehta, 1995).

Taylor's hypothesis is limited by the fact that the turbulent fluctuations also evolve in time, which means they usually cannot be regarded as frozen-in. This limitation becomes especially apparent when the turbulent fluctuations are comparable to the mean flow, such as at high turbulence intensities. Moreover, the fluctuations of the velocity field contain largescale variations, sometimes termed random sweeping velocity (Kraichnan, 1964; Tennekes, 1975), which are difficult to discriminate from the mean velocity due to their large-scale nature. The aim of this paper is to focus on the latter effect and to quantify the influence of these large-scale flow variations on the energy spectrum as it is usually measured in experiments.

This idea has been discussed in prior literature, for example by Lumley (1965) who studied the effects of random sweeping by keeping only the first two terms in the series expansion of the characteristic function of the largescale velocity fluctuations. His work later was picked up by Wyngaard and Clifford (1977) and compared to the case of a Gaussian large-scale random sweeping velocity. They found that the Gaussian random sweeping assumption agrees well with Lumley's two-term expansion for low to moderate turbulence intensities. Based on their model calculations they were able to show that random sweeping effects lead to systematic deviations in many statistical quantities inferred from single-point measurements using Taylor's hypothesis, including the spectral energy levels.

In this note, we make use of a simple theoretical model recently introduced for the wavenumber-frequency spectrum by Wilczek and Narita (2012). This model spectrum can be regarded as the result of a generalized Taylor's hypothesis, which takes into account the advection of turbulent fluctuations not only by a mean flow, but also by a random largescale velocity field and is essentially based on Kraichnan's ideas of random sweeping (Kraichnan, 1964). Based on the same mathematical approach also used by Wyngaard and Clifford for their model calculations, our results are in line with their findings. Here, we will however focus the discussion on the impact of large-scale random sweeping on the determination of the Kolmogorov constant. In particular, we obtain a closed-form analytical expression that quantifies the effect of large-scale random sweeping on the Kolmogorov constant determined using Taylor's hypothesis.

The procedure is the following: given an energy spectrum function in wavenumber space, our theoretical model makes an estimate for the wavenumber-frequency spectrum parametrized by the mean and the sweeping velocity. This wavenumber-frequency spectrum serves as an "ideal" reference spectrum, which then can be reduced to the frequency spectrum. In the next step, Taylor's hypothesis, as used in experiments, is applied to the frequency spectrum to obtain an expression for the energy spectrum function in wavenumber space, here called the Taylor spectrum. By comparing this approximate spectrum to the "true" Kolmogorov energy spectrum function, the influence of large-scale flow variations can be systematically tested. It especially allows discussing corrections to the experimental measurement of the Kolmogorov constant due to random sweeping effects.

\section{Wavenumber-frequency spectrum}

As detailed in Wilczek and Narita (2012), we make a number of simplifying assumptions to obtain an analytical model for the wavenumber-frequency spectrum. We assume that the large-scale random sweeping velocity and the small-scale velocity fluctuations are statistically independent initially. Furthermore, we assume the random advection to be isotropic and Gaussian distributed and the small scales to be isotropic with a given energy spectrum function. We then essentially consider a linear advection problem in which the turbulent fluctuations are advected by a mean velocity $U$ (assumed in $z$ direction) and the random advection velocity (characterized by the rms of each component, $V$ ). This eventually leads to an analytical expression for the energy $E(\boldsymbol{k}, \omega) \mathrm{d} \boldsymbol{k} \mathrm{d} \omega$ contained in an infinitesimal wave vector and frequency volume. In this model, the mean velocity leads to a Doppler shift of frequencies, whereas the random sweeping leads to a Doppler broadening. Specifically, the expression obtained 
is a product of a wavenumber spectrum with a Gaussian frequency distribution:

$$
\begin{aligned}
E(\boldsymbol{k}, \omega) \mathrm{d} \boldsymbol{k} \mathrm{d} \omega= & \frac{E(k)}{4 \pi k^{2} \sqrt{2 \pi k^{2} V^{2}}} \\
& \exp \left[-\frac{\left(\omega-k_{z} U\right)^{2}}{2 k^{2} V^{2}}\right] \mathrm{d} \boldsymbol{k} \mathrm{d} \omega .
\end{aligned}
$$

Here, $E(k)$ is the energy spectrum function of the smallscale velocity fluctuations. In the following, we will restrict ourselves to a power-law spectrum, which follows Kolmogorov's phenomenology, cf. Eq. (1). The mean of the Gaussian distribution $k_{z} U$ describes the Doppler shift, whereas the standard deviation $k V$ describes the Doppler broadening. Note that both effects become more important with increasing wavenumbers.

It is worthwhile to note that in the limit of vanishing sweeping velocity, Taylor's hypothesis again becomes valid. This can be seen by

$\lim _{V \rightarrow 0} E(\boldsymbol{k}, \omega) \mathrm{d} \boldsymbol{k} \mathrm{d} \omega=\frac{E(k)}{4 \pi k^{2}} \delta\left(\omega-k_{z} U\right) \mathrm{d} \boldsymbol{k} \mathrm{d} \omega$,

where the delta function simply indicates that the frequency can be relabeled with the streamwise wavenumber. As the standard deviation of the Gaussian distribution of the model spectrum is $k V$, the rate of convergence to a delta function decreases with increasing $k$.

By integrating the model (Eq. 2) over the wave vector domain, the energy in the frequency domain can be obtained as (Wilczek and Narita, 2012)

$E(\omega) \mathrm{d} \omega=C_{\mathrm{F}}(U, V) C_{\mathrm{K}} \varepsilon^{2 / 3} \omega^{-5 / 3} \mathrm{~d} \omega$,

where $C_{\mathrm{F}}$ denotes the coefficient

$C_{\mathrm{F}}(U, V)=\int_{0}^{\infty} \mathrm{d} x\left[\operatorname{erf}\left(\frac{x+U}{\sqrt{2} V}\right)-\operatorname{erf}\left(\frac{x-U}{\sqrt{2} V}\right)\right] \frac{x^{2 / 3}}{2 U}$.

Here we have assumed an infinitely extended inertial range. Note that while the frequency $\omega$ in Eq. (2) can take both positive and negative values, we consider only positive frequencies in Eq. (4) since positive and negative frequencies cannot be discriminated by a single-point measurement. Consequently, a factor of two is included into Eq. (5) in order to conserve the total energy. Given an Eulerian wavenumber spectrum with index $-5 / 3$, also the Eulerian frequency spectrum will exhibit this power-law index, independent of mean flow and sweeping velocity. The prefactor, however, depends on $U$ and $V$. This implies that the observation of a frequency spectrum consistent with Kolmogorov's phenomenology does not guarantee the validity of Taylor's hypothesis.

\section{Implications for the measurement of the Kolmogorov constant}

We now come to the main part of this paper and discuss the implications of our model on the measurement of the Kolmogorov constant. To this end, we derive an expression for the energy spectrum function obtained with the help of Taylor's hypothesis. This will be referred to as the Taylor energy spectrum function, which then can be compared to the Kolmogorov energy spectrum function. We start by stressing that the quantity $E(\omega) \mathrm{d} \omega$ represents the energy contained in an infinitesimal frequency interval $\mathrm{d} \omega$. This quantity is experimentally accessible by measuring time series of all velocity components, for example by anemometry with an X-wire configuration. To apply Taylor's hypothesis, the frequency is simply related to the wavenumber in the streamwise direction by $\omega=k_{z} U$, which upon insertion into Eq. (4) leads to

$$
\begin{aligned}
\widetilde{E}\left(k_{z}\right) \mathrm{d} k_{z} & =E(\omega) \frac{\mathrm{d} \omega}{\mathrm{d} k_{z}} \mathrm{~d} k_{z} \\
& =C_{\mathrm{F}}(U, V) U^{-2 / 3} C_{\mathrm{K}} \varepsilon^{2 / 3} k_{z}^{-5 / 3} \mathrm{~d} k_{z} .
\end{aligned}
$$

This spectrum describes the energy density of the turbulent fluctuations resolved with respect to the streamwise wavenumber $k_{z}$ and thus is related to the one-dimensional spectra (Pope, 2000)

$E_{i j}\left(k_{z}\right)=\frac{1}{\pi} \int_{-\infty}^{\infty} \mathrm{d} z\left\langle u_{i}(\boldsymbol{x}) u_{j}\left(\boldsymbol{x}+z \boldsymbol{e}_{z}\right)\right\rangle \exp \left[-i k_{z} z\right]$

by

$\widetilde{E}\left(k_{z}\right)=\frac{1}{2} E_{i i}\left(k_{z}\right)$.

For power-law spectra of Kolmogorov type, as considered here, a simple relation between the one-dimensional spectra and the energy spectrum function exists in the inertial range (Pope, 2000),

$\frac{1}{2} E_{i i}(k)=\frac{3}{5} E(k)$.

Together with Eq. (6), this leads to the Taylor energy spectrum function:

$E_{\mathrm{T}}(k)=C_{\mathrm{T}}(U, V) C_{\mathrm{K}} \varepsilon^{2 / 3} k^{-5 / 3}$

with

$C_{\mathrm{T}}(U, V)=\frac{5}{3} U^{-2 / 3} C_{\mathrm{F}}(U, V)$,

where the subscript $\mathrm{T}$ is introduced to discriminate the energy spectrum function based on our model under application of Taylor's hypothesis from the "true" Kolmogorov energy spectrum function. 
The interesting point about this result is that the energy spectrum measured with Taylor's hypothesis takes the form of the original Kolmogorov energy spectrum function (Eq. 1) multiplied by a factor that depends on the mean and random sweeping velocities. $C_{\mathrm{T}}(U, V)$ is a non-dimensional prefactor that depends only on the ratio $\xi=V / U$. $\xi$ can be identified with the turbulence intensity in good approximation, because the kinetic energy in the small-scale velocity field is small compared to the fluctuations of the random sweeping field. To explicitly see that, we substitute Eq. (5) into Eq. (11) and obtain

$C_{\mathrm{T}}(\xi)=\frac{5}{6} \int_{0}^{\infty} \mathrm{d} y\left[\operatorname{erf}\left(\frac{y+1}{\sqrt{2} \xi}\right)-\operatorname{erf}\left(\frac{y-1}{\sqrt{2} \xi}\right)\right] y^{2 / 3}$.

This result stresses the importance of the turbulence intensity for the validity of Taylor's hypothesis. In the limit of vanishing turbulence intensity (corresponding to $\xi \rightarrow 0$ ) the "true" Kolmogorov energy spectrum function has to be recovered. In this case the error functions in the integrand reduce to sharp Heaviside cutoffs, which leads to

$\lim _{\xi \rightarrow 0} C_{\mathrm{T}}(\xi)=\frac{5}{3} \int_{0}^{1} \mathrm{~d} y y^{2 / 3}=1$.

For non-vanishing turbulence intensities, however, these cutoffs are smoothed out. A closer inspection of the expression shows that $C_{\mathrm{T}} \geq 1$. This can be seen by appreciating that the error-function term represents a bump-shaped function which preserves its area irrespective of the turbulence intensity. Compared to the zero-turbulence intensity case, the amplitude of the bump is decreased for $y<1$, but almost symmetrically we now get contributions for $y>1$. As this error function term in the integrand is weighed by the monotonically increasing $y^{2 / 3}$, the correction has to be positive and increasing with the turbulence intensity.

A graphical evaluation of the coefficient $C_{\mathrm{T}}$ as a function of turbulence intensity is presented in Fig. 1. It is evident that the influence for moderate turbulence intensities below $25 \%$, as they are usually met in experimental setups, is quite small. For example, in conventional passive grid turbulence in a wind tunnel, the turbulence intensity $\xi$ is typically below $5 \%$. Hence the correction to $C_{\mathrm{K}}$ expected from our theory is negligible. In more recent active grid turbulence in a wind tunnel, the turbulence intensity is usually below $20 \%$, which leads to corrections of around $2 \%$ or less. For turbulent jets, $\xi$ in the centerline can reach about $25 \%$. Even in this case the correction to $C_{\mathrm{K}}$ is still small, approximately $3.5 \%$ only. If, however, the large-scale fluctuations exceed the mean flow, significant errors occur.

As summarized in Sreenivasan (1995), experimentally measured $C_{\mathrm{K}}$ for $50<R_{\lambda} \lesssim 2 \times 10^{4}$ lie within the range $1.62 \pm 0.17$. These values are in general agreement with DNS results, but the scatter of data is significant. Sreenivasan

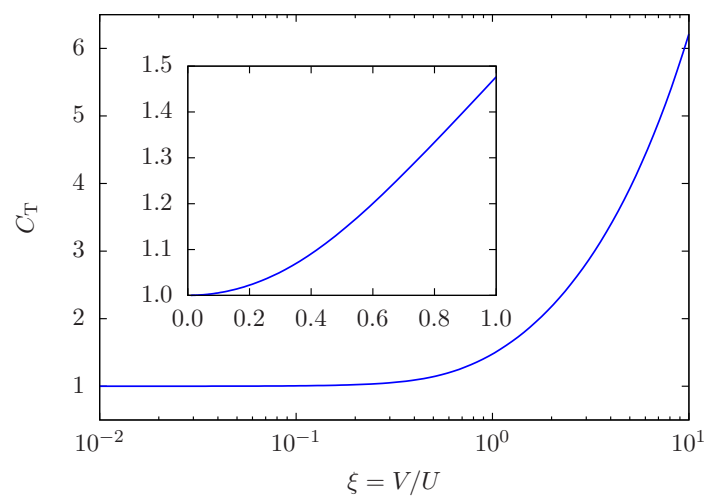

Figure 1. Coefficient $C_{\mathrm{T}}$ of the Taylor spectrum (Eq. 10) as a function of the (approximate) turbulence intensity $\xi=\frac{V}{U}$.

(1995) argued that neither the stability nor the large-scale shear in the atmospheric surface layer caused large variations of $C_{\mathrm{K}}$; each of the two mechanisms affects $C_{\mathrm{K}}$ by a few percent. The results discussed here, in line with those of Wyngaard and Clifford, provide an additional source which adds to the experimental scatter in measured $C_{\mathrm{K}}$ values in geophysical flows, which are featured with strong fluctuations at large scales.

It would also be interesting to extend our analysis to plasma turbulence, in which a $-5 / 3$ spectrum in the frequency domain has been reported by various in situ spacecraft observations of the magnetic field in solar wind turbulence, for example, ranging over nearly three orders of magnitude as measured by the WIND spacecraft (Podesta et al., 2007). Naively speaking, the use of Taylor's hypothesis seems to be justified in this case since the fluctuation of the flow velocity is of the order of $10 \%$ of the mean flow (about $400 \mathrm{~km} \mathrm{~s}^{-1}$ ). However, the magnetic field fluctuation may reach $100 \%$ of the mean field (which is in the range 1 to $10 \mathrm{nT}$ ). Thus the effect of the Alfvén waves needs to be included for a quantitative estimate of Taylor's hypothesis in the solar wind.

\section{Conclusions}

To summarize, we have used a simple model for the wavenumber-frequency spectrum to systematically quantify the influence of large-scale sweeping velocities on wavenumber spectra that are experimentally accessible using Taylor's hypothesis. While the application of Taylor's hypothesis for power-law spectra yields the correct scaling behavior, we have found that random sweeping effects lead to a systematic overestimation of the Kolmogorov constant. For moderate turbulence intensities, as usually met in laboratory experiments, the corrections are of the order of a few percent. In geophysical and astrophysical situations, where the flow is usually is not as well controlled as in the laboratory, the 
turbulence intensities can reach high levels where significant corrections to the measurement have to be taken into account.

Acknowledgements. M. Wilczek acknowledges funding by DFG WI 3544/2-1. H. Xu gratefully acknowledges partial support from DFG through project A7 in SFB 963 AstroFIT. The work is also supported by FP7 313038/STORM.

Edited by: B. Tsurutani

Reviewed by: O. Verkhoglyadova and another anonymous referee

\section{References}

Li, B., Murthi, A., Bowman, K. P., North, G. R., Genton, M. G., and Sherman, M.: Statistical tests of Taylor's hypothesis: an introduction to precipitation fields, J. Hydrometeorol., 10, 254-265, 2009.

Castro, J. J., Carsteanu, A. A., and Fuentes, J. D.: On the phenomenology underlying Taylor's hypothesis in atmospheric turbulence, Revista Mexicana de Física, 57, 60-64, 2011.

Kolmogorov, A. N.: The local structure of turbulence in incompressible viscous fluid for very large Reynolds number, Dokl. Akad. Nauk. SSSR, 30, 299-303, 1941.

Kraichnan, R. H.: Kolmogorov's hypothesis and Eulerian turbulence theory, Phys. Fluids, 7, 1723-1734, 1964.

Lappe, U. O. and Davidson, B.: On the range of validity of Taylor's hypothesis and the Kolmogoroff spectral law, J. Atmos. Sci., 20, 569-576, 1963.

LeBoeuf, R. L. and Mehta, R. D.: On using Taylor's hypothesis for three-dimensional mixing layers, Phys. Fluids, 7, 1516-1518, 1995.
Lumley, J. L.: Interpretation of time spectra measured in highintensity shear flows, Phys. Fluids, 8, 1056-1062, 1965.

MacMahan, J., Reniers, A., Ashley, W., and Thornton, E.: Frequency-wavenumber velocity spectra, Taylor's hypothesis, and length scales in a natural gravel bed river, Water Resource Res., 48, W09548, doi:10.1029/2011WR011709, 2012.

Mizuno, T. and Panofsky, H. A.: The validity of Taylor's hypothesis in the atmospheric surface layer, Bound.-Lay. Meteorol., 9, 375380, 1975.

Podesta, J. J., Roberts, D. A., and Goldstein, M. L.: Spectral exponents of kinetic and magnetic energy spectra in solar wind turbulence, Astrophys. J., 664, 543-548, 2007.

Pope, S. B.: Turbulent Flows, Cambridge Univ. Press, Cambridge, UK, 2000.

Sreenivasan, K. R.: On the universality of the Kolmogorov constant, Phys. Fluids, 7, 2778-2784, 1995.

Taylor, G. I.: The spectrum of turbulence, P. Roy. Soc. Lond. A, 164, 476-490, 1938.

Tennekes, H.: Eulerian and Lagrangian time microscales in isotropic turbulence, J. Fluid Mech., 67, 561-567, 1975.

Uddin, A. K. M., Marusic, A. E. P., and Marusic, I.: On the validity of Taylor's hypothesis in wall turbulence, J. Mech. Eng. Res. Dev., 19-20, 57-66, 1997.

Wilczek, M. and Narita, Y.: Wave-number-frequency spectrum for turbulence from a random sweeping hypothesis with mean flow, Phys. Rev. E, 86, 066308, doi:10.1103/PhysRevE.86.066308, 2012.

Wyngaard, J. C. and Clifford, S. F.: Taylor's hypothesis and highfrequency turbulence spectra, J. Atmos. Sci., 34, 922-929, 1977. 